\title{
Using Importance Sampling for Bayesian Feature Space Filtering
}

\author{
Anders Brun ${ }^{1,4}$, Björn Svensson ${ }^{1,4}$, Carl-Fredrik Westin ${ }^{2}$, Magnus Herberthson ${ }^{3}$, \\ Andreas Wrangsjö ${ }^{1,4}$, and Hans Knutsson ${ }^{1,4}$ \\ ${ }^{1}$ Department of Biomedical Engineering, Linköping University, Sweden \\ andbraimt.liu.se \\ 2 Department of Mathematics, Linköping University, Sweden \\ ${ }^{3}$ Laboratory of Mathematics in Imaging, Harvard Medical School, Boston, USA \\ ${ }^{4}$ Center for Medical Image Science and Visualization, Linköping University, Sweden
}

\begin{abstract}
We present a one-pass framework for filtering vector-valued images and unordered sets of data points in an $N$-dimensional feature space. It is based on a local Bayesian framework, previously developed for scalar images, where estimates are computed using expectation values and histograms. In this paper we extended this framework to handle $N$-dimensional data. To avoid the curse of dimensionality, it uses importance sampling instead of histograms to represent probability density functions. In this novel computational framework we are able to efficiently filter both vector-valued images and data, similar to e.g. the wellknown bilateral, median and mean shift filters.
\end{abstract}

\section{Introduction}

In this paper we present a method for filtering of vector-valued images, $x(q) \in \mathbb{V}=\mathbb{R}^{n}$, where $\mathbb{V}$ is a feature vector space such as the RGB color space. For the purposes of this paper, $q$ is a point in a spatial vector space, $q \in \mathbb{U}=\mathbb{R}^{m}$, e.g. $q \in \mathbb{R}^{2}$ for images. It is however easy to extend this filtering to a curved $m$-dimensional manifold, $q \in M$. We also show how a slight modification can generalize this method to be used for filtering unordered sets of data points in a feature space, $\left\{x_{i}\right\} \in \mathbb{V}=\mathbb{R}^{n}$.

The proposed method is inspired by previous work by Wrangsjö et al. [17], a local Bayesian framework for image denoising of scalar-valued images. That method was based on a computational framework involving histograms, which made it slow and nearly impossible to use for vector-valued images. In this paper we propose the novel use of a Monte Carlo method called importance sampling to overcome this difficulty. It makes this particular kind of Bayesian filtering feasible for vector-valued images and data.

\section{Previous Work}

In [17] the proposed filter is related to bilateral filters [6]10[15]16]. Other filters operating on local neighborhoods in images with similar characteristics include mean shiftfiltering [4], median filters [2], total variation filters [14], diffusion based noise reduction [3]13] and steerable filters [59]. Several of these filters are compared in [12]. 


\section{The Bayesian Method}

The method is founded on Bayesian theory and for this reason the a posteriori probability distribution function, $p_{S \mid X=x}(s)$, is important. If we let $s$ be the true value and $x$ be the measured value which is corrupted by noise then

$$
p_{S \mid X=x}(s)=\frac{p_{X \mid S=s}(x) p_{S}(s)}{p_{X}(x)} .
$$

In order to derive an estimate $\hat{s}$ of the true signal $s$ from the above formula, the conditional expectation value of $s$ may be calculated,

$$
\hat{s}=\int_{s \in \mathbb{V}} s p_{S \mid X=x}(s) d s=\left.E[S]\right|_{X=x} .
$$

This is the Minimum Mean Squared Error estimate, which can be calculated if the different probability distributions are modelled appropriately.

\subsection{Noise Models}

The modelling of noise, how measurements are related to the true signal value, is important. For the general case, the conditional probability $p_{X \mid S=s}(x)$ need to be known and in many applications this is not a problem. For the special case of additive noise, $X=S+N$, where $N$ can belong to e.g. a Gaussian or super-Gaussian distribution, some simplifications can be made,

$$
\begin{aligned}
p_{X \mid S=s}(x) & =\int_{t \in \mathbb{V}} \delta(x-t-s) p_{N}(t) d t \\
& =p_{N}(x-s) .
\end{aligned}
$$

For some important special cases, in particular Rician noise which is present in Magnetic Resonance (MR) images, the additive model is however not valid unless the noise is approximated using a Gaussian distribution.

It should also be mentioned that the present method can only handle cases where the measurements can be considered to be independent and identically distributed (i.i.d.). This makes it difficult to handle e.g. speckle noise in ultrasound images efficiently.

\subsection{Signal Models for Images}

Most of the power of the method proposed in [17] is embedded in the a priori p.d.f., $p_{S}(s)$, which is derived from a local neighborhood around the pixel which is to be estimated. Without knowledge of the exact distribution, a kernel (Parsen window) estimate of $p_{X}(x)$ is used to model a suitable local prior:

$$
\begin{aligned}
p_{S}(s) & =C_{0}\left[\sum_{i} b_{v}\left(x_{i}-s\right) b_{s}\left(q_{0}-q_{i}\right)\right]^{\alpha} \\
& \approx C_{0} p_{X}(s)^{\alpha}
\end{aligned}
$$


where $b_{v}(\cdot)$ is the kernel used to approximate density in $\mathbb{V}$, e.g. a Gaussian, and $b_{s}(\cdot)$ is a similar spatial weight which is used to favor samples which are close to $q_{0}$, the position of the pixel to be estimated. The normalizing constant $C$ has no effect on the estimate, but the exponent $\alpha \geq 1$ make the histogram sharper and a higher value of $\alpha$ promote a harder bias towards the most probable mode in the distribution $p_{X}(x)$. This local modelling is ad hoc, but has proven to work surprisingly well in practice.

\subsection{Signal Models for N-D Data Sets}

For unordered data we need to slightly modify this approach. We propose a similar way to model the a priori distribution for unordered data, the difference being the lack of a spatial weight.

$$
\begin{aligned}
p_{S}(s) & =C_{1}\left[\sum_{i} b_{v}\left(x_{i}-s\right)\right]^{\alpha} \\
& \approx C_{2} p_{X}(s)^{\alpha}
\end{aligned}
$$

\subsection{Estimation}

In the original approach for scalar images, histograms were used to estimate the $a$ priori density function. Since the continuous integrals could not be evaluated exactly, all integrations were performed numerically in this way. In this paper we instead propose a solution based on importance sampling to calculate Eq. 1. more efficiently.

\section{Importance Sampling}

In the original approach for scalar-valued images, discretized histograms were used to estimate the a priori density function in the numerical calculation of the estimate given by Eq.1 This turned out to be infeasible for vector-valued images.

It is evident that the integral in Eq. 1 can be evaluated using Monte Carlo, by drawing samples $s_{i}$ from $p_{S \mid X=x}(s)$ and calculate the expectation value numerically. This correspond to the upper left illustration in Fig. 1. Sampling from a distribution can however be tricky and we will now introduce the concepts proper samples and importance sampling which will give us some freedom.

\subsection{Proper Samples}

We define the following [178[1]]. A set of weighted random samples $\left\{z_{i}, w_{i}\right\}, z_{i} \in$ $p_{Z}$, is called proper with respect to a distribution $p_{X}$ if for any square integrable function $h(\cdot)$,

$$
\begin{array}{r}
E\left[w_{i} h\left(z_{i}\right)\right]=c E\left[h\left(x_{i}\right)\right] \Leftrightarrow \\
\int w(y) h(y) p_{Z}(y) d y=c \int h(y) p_{X}(y) d y
\end{array}
$$



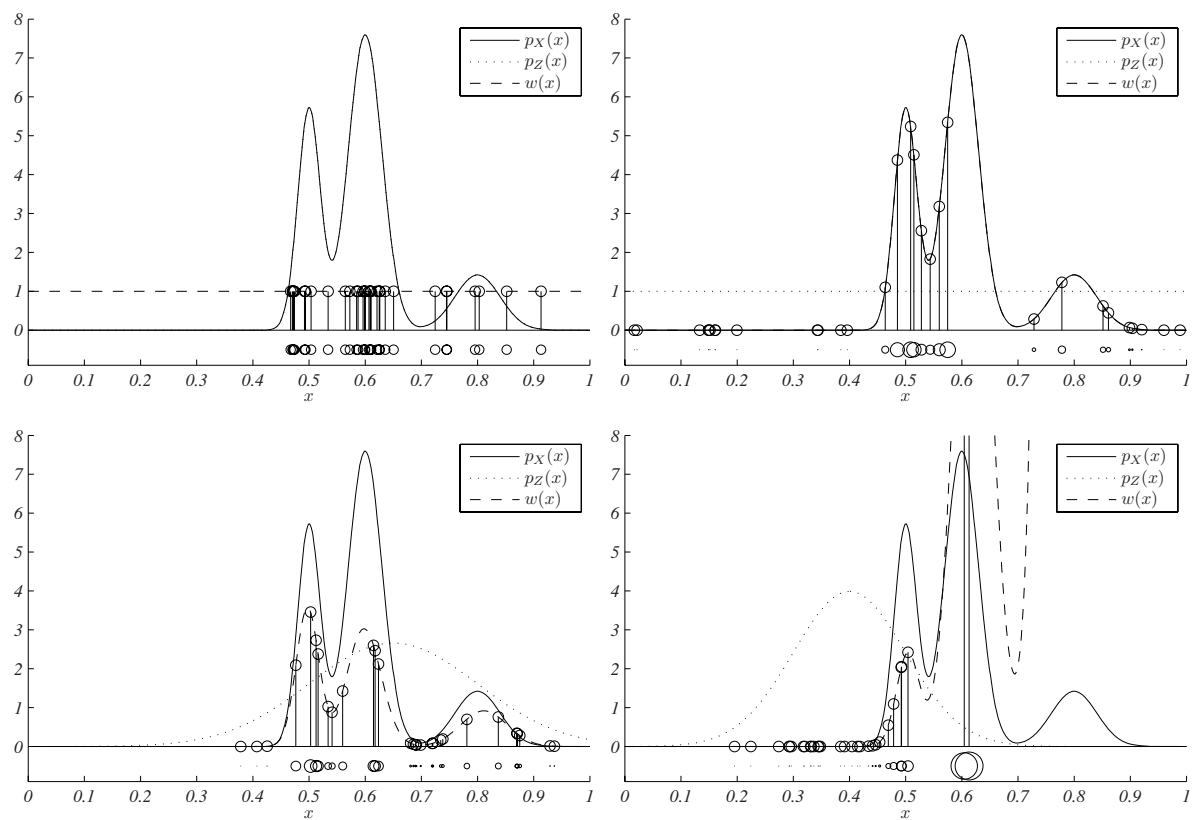

Fig. 1. Examples of sampling. T-L: Sampling from $p_{X}(x)$. T-R: Sampling from a uniform distribution, weighting with $w_{i}=p_{X}\left(x_{i}\right)$. B-L: Sampling using a Gaussian as a trial distribution. B-R: Sampling using a not so suitable Gaussian trial distribution.

for some constant $c$. Since this should be valid for any $h(\cdot), w(y)=c p_{X}(y) / p_{Z}(y)$, and

$$
\begin{aligned}
\int c p_{X}(y) d y & =\int w(y) p_{Z}(y) d y \\
c & =E\left[w\left(z_{i}\right)\right] .
\end{aligned}
$$

\subsection{Importance Sampling}

The notion of proper samples now allow us to numerically calculate the expectation value of a distribution $p_{X}$ using $M$ samples from a trial distribution $p_{Z}$,

$$
\begin{aligned}
E\left[h\left(x_{i}\right)\right] & =\frac{1}{c} E\left[w_{i} h\left(z_{i}\right)\right] \\
& \approx \frac{1}{\sum_{i}^{M} w_{i}} \sum_{i}^{M} w_{i} h\left(z_{i}\right) .
\end{aligned}
$$

This is how expectation values are calculated in importance sampling. It can be used when sampling from $p_{X}$ is difficult but sampling from $p_{Z}$ is easy. This is the case if the trial distribution $p_{Z}$ is e.g. a uniform distribution, a Gaussian or a mixture of Gaussians. For us it means that we can evaluate the integral in Eq. 1 by sampling from another 
distribution $p_{Z}$, if we choose the weights $w_{i}$ appropriately. For the application at hand, we choose a trial distribution which is similar to the distribution of pixel-values found in the window defined by $b_{s}(\cdot)$.

In figure 1 some examples of proper sampling are shown. Note in particular that even though evaluation using importance sampling theoretically converge to the correct expectation value when $M \rightarrow \infty$, an unsuitable choice of trial distribution may give very slow convergence. Generically, the weight $w_{i}$ for a sample $z_{i}$ should be chosen so that $w_{i}=p_{X}\left(z_{i}\right) / p_{Z}\left(z_{i}\right)$. If these weights grow very large, it is an indication that convergence towards the true expectation value will be slow.

\section{Implementation}

The Bayesian feature space filtering method was implemented in Matlab and tested using various choices of trial functions. Two variants were derived, one for vectorvalued images and one for unordered sets of data.

\subsection{Vector-Valued Images}

The filter was evaluated for each pixel in the image, $x_{i}$ being the values of the pixels in a neighborhood large enough to fit the spatial weight function $b_{s}(q)$. In the following, $x_{0}$ is the measured value in the pixel to be estimated, located at position $q_{0}$. The function $b_{v}(x)$ is an isotropic Gaussian distribution with zero mean and standard deviation $\sigma_{v}$, corresponding to a kernel in the feature space used in the density estimation. In the spatial domain $b_{s}(q)$ is an isotropic Gaussian weight function with standard deviation $\sigma_{s}$. The noise of the pixel to be estimated, $x_{0}$, is modelled using $p_{X \mid S=z}\left(x_{0}\right)$, which is also an isotropic Gaussian distribution with standard deviation $\sigma_{n}$. The conditional expectation value of $S$ can now be expressed using the stochastic variable $Z$ which is distributed according to the trial distribution.

$$
\begin{aligned}
\bar{s} & =\left.E[S]\right|_{X=x_{0}} \\
& =\int_{s \in U} s p_{S \mid X=x_{0}}(s) d s \\
& =E[Z w(Z)] / E[w(Z)]
\end{aligned}
$$

is approximated for a finite number of samples by

$$
\hat{s}=\frac{1}{\sum_{i=1}^{M} w\left(z_{i}\right)} \sum_{i=1}^{M} z_{i} w\left(z_{i}\right) .
$$

The weight which should be used to guarantee proper samples is

$$
\begin{aligned}
w(z) & =\frac{p_{S \mid X=x_{0}}(z)}{p_{Z}(z)} \\
& =\frac{p_{X \mid S=z}\left(x_{0}\right) p_{S}(z)}{p_{Z}(z) p_{X}\left(x_{0}\right)},
\end{aligned}
$$



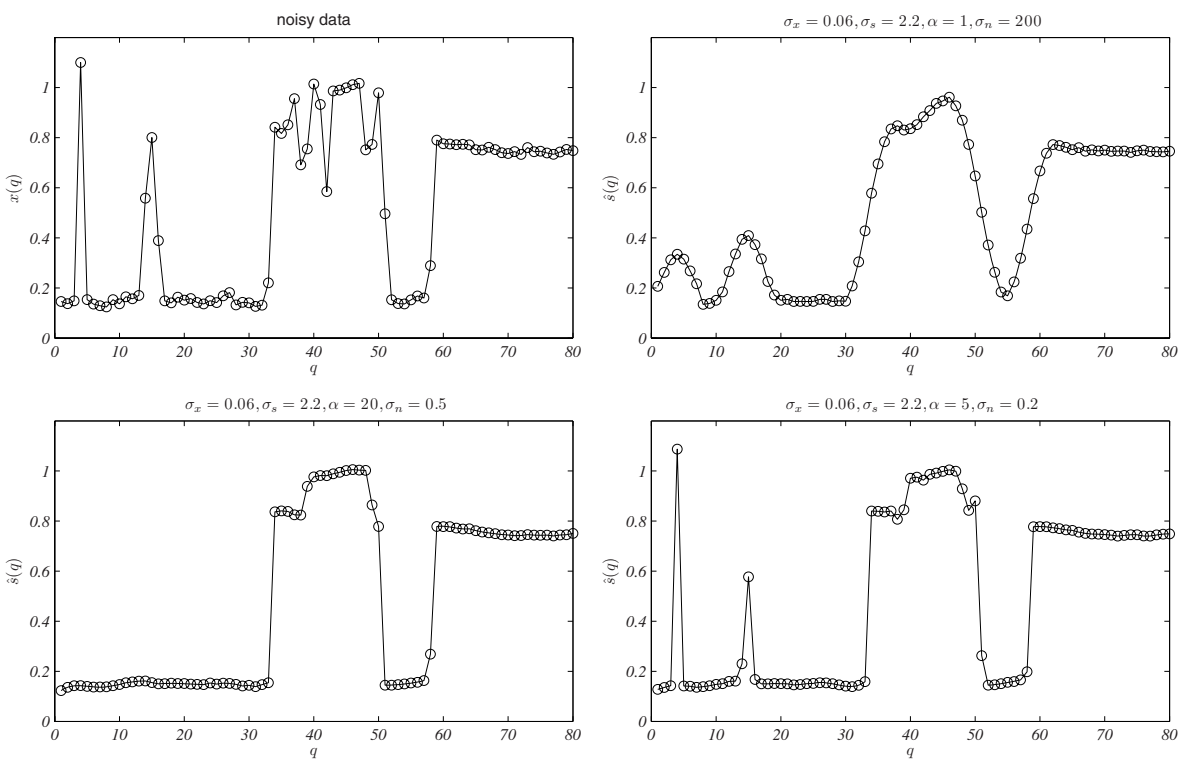

Fig. 2. Filtering a 1-D scalar signal. Parameters are shown in the figure.

where $p_{X}\left(x_{0}\right)$ is a consequence of Bayes rule in the derivation above, but in practice has no effect on the estimate. The prior $p_{S}(z)$ is modelled using Eq. 2 and the trial distribution used in the sampling is a mixture of Gaussians,

$$
p_{Z}(z)=\frac{1}{C_{3}} \sum_{i} b_{v}\left(x_{i}-z\right)
$$

which is fairly easy to sample from. In general the choice of trial distribution is very important when implementing importance sampling. In our experiments we found that this local estimate of $p_{X}$ worked well in this particular application. Generically this distribution will contain the same modes and have the same support as the a posteriori distribution we are interested in. Ignoring all constants, the weights can be calculated,

$$
w(z)=p_{X \mid S=z}\left(x_{0}\right)\left[\sum_{i} b_{v}\left(x_{i}-z\right) b_{s}\left(q_{0}-q_{i}\right)\right]^{\alpha} / \sum_{i} b_{v}\left(x_{i}-z\right) .
$$

A non-stochastic alternative would have been to use the samples $x_{i}$ themselves, in the neighborhood of $x_{0}$, as samples $z_{i}$ and use the estimate of $p_{X}$ in the neighborhood to approximate their probability density function. We implemented this variant and it worked well, but for the experiments on images reported in this paper we have actually used true importance sampling, with a neighborhood of $5 \times 5$ pixels and 125 samples $z_{i}$ from the trial distribution $Z$ in each neighborhood. 

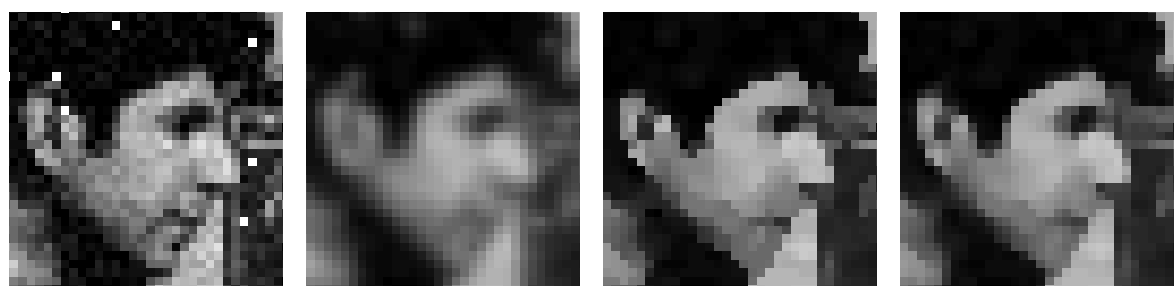

Fig. 3. Filtering a noisy 2-D scalar image with outliers. Left-Right: Noisy data. $\left[\sigma_{v}=0.04\right.$, $\left.\sigma_{n}=100, \sigma_{s}=1.0, \alpha=1\right] .\left[\sigma_{v}=0.04, \sigma_{n}=0.5, \sigma_{s}=1.0, \alpha=20\right] .\left[\sigma_{v}=0.04\right.$, $\sigma_{n}=0.5, \sigma_{s}=1.0, \alpha=5$.]
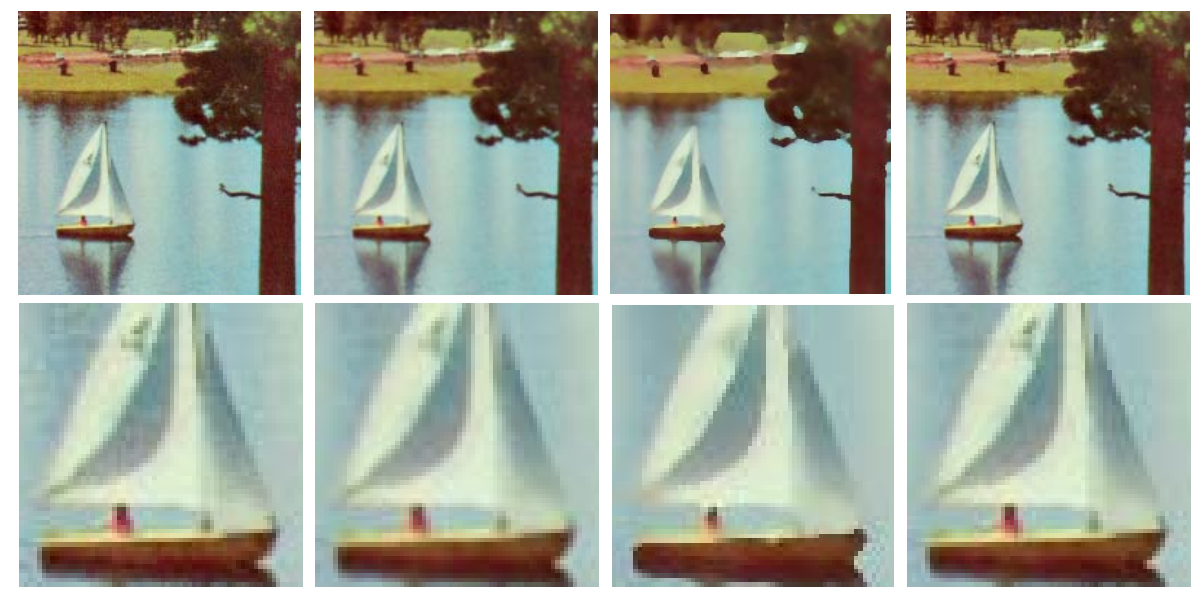

Fig. 4. Filtering a noisy 2-D RGB image. Left-Right: Noisy data. $\left[\sigma_{v}=0.04, \sigma_{n}=100, \sigma_{s}=\right.$ $0.8, \alpha=2] .\left[\sigma_{v}=0.14, \sigma_{n}=0.6, \sigma_{s}=2.0, \alpha=20\right] .\left[\sigma_{v}=0.04, \sigma_{n}=0.2, \sigma_{s}=0.8\right.$, $\alpha=6]$.

\subsection{Unordered N-D Data}

For an unordered set of $N$-dimensional data, we use the prior defined in Eq. 4 , i.e. we regard all elements in $\left\{x_{i}\right\}$ as "neighbors" to the point $x_{0}$ to be estimated, and repeat this procedure for each choice of $x_{0} \in\left\{x_{i}\right\}$. The trial distribution from Eq. 6 is used and the lack of spatial weighting allow us to simplify the weight function,

$$
w(z)=p_{X \mid S=z}\left(x_{0}\right)\left[\sum_{i} b_{v}\left(x_{i}-z\right)\right]^{\alpha-1} .
$$

Observing that the trial distribution used here is essentially the same as the distribution of points in $\left\{x_{i}\right\}$, we use approximated importance sampling in the implementation. This means that instead of sampling from the true trial distribution, we choose $z_{i}=x_{i}$. 

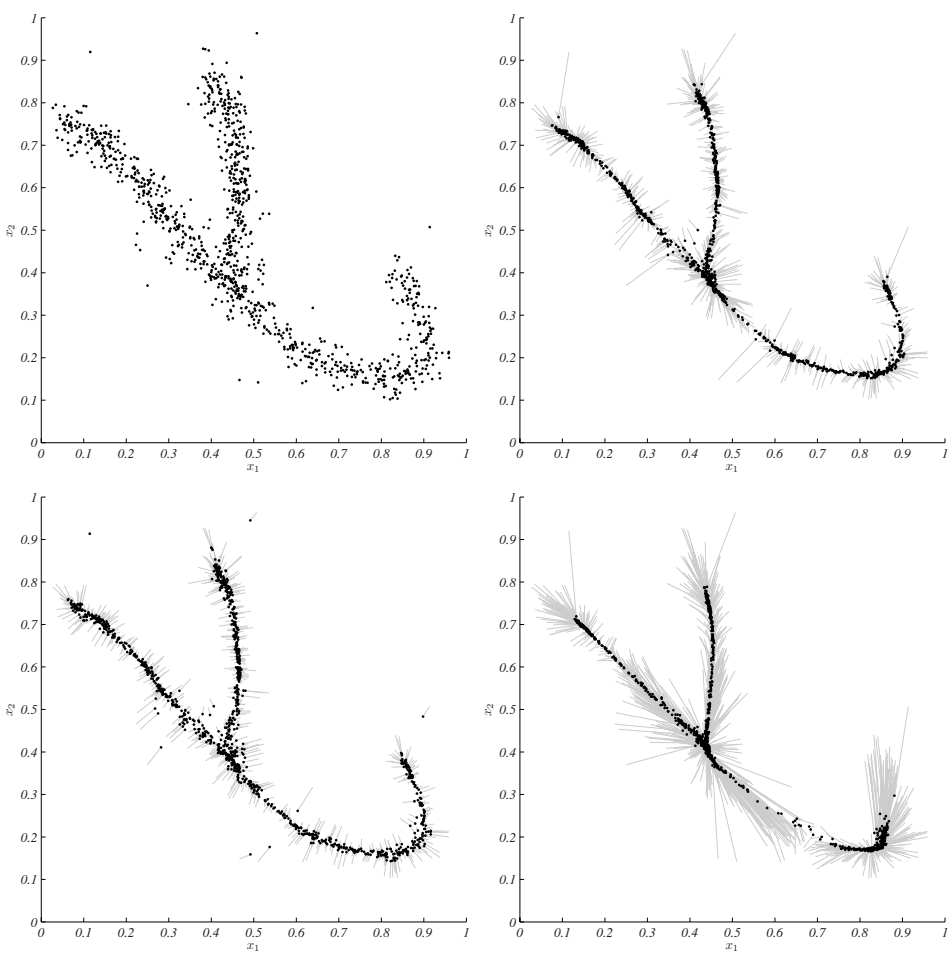

Fig. 5. Filtering unordered 2-D. The data is a 1-D "manifold" embedded in 2-D, corrupted by noise and outliers. The gray arrows show the how each point has moved in the resulting image. T-L: Noisy data. T-R: $\sigma_{v}=0.05, \sigma_{n}=0.05, \alpha=6$. B-L: $\sigma_{v}=0.15, \sigma_{n}=0.06, \alpha=1$. B-R: $\sigma_{v}=0.1, \sigma_{n}=0.08, \alpha=20$.

This deterministic procedure turned out to give very similar results to true importance sampling when the number of data points was large enough.

\section{Experiments}

Some experiments are included to demonstrate the proposed method.

\subsection{Scalar Signals}

Experiments in Fig. 2 shows a simple example of filtering a 1-D signal. In Fig. 3 the method was tried out on a scalar image. These two experiments were included mainly to illustrate the behavior of the filter and show that it is similar to the previous filter proposed in [17].

\subsection{Vector-Valued Signals}

Next the filter was tested on 2D color images, encoded as pixels with RGB color vectors. The parameters of the filters were tuned manually and Fig. 4 show both good and bad results. 


\subsection{Unordered N-D Data}

The filter was then tested on unordered 2-D and 3-D data, see Fig. 5 and Fig. 6 The data points in Fig. 6 were derived from the RGB-values of the boat image in Fig. 4.
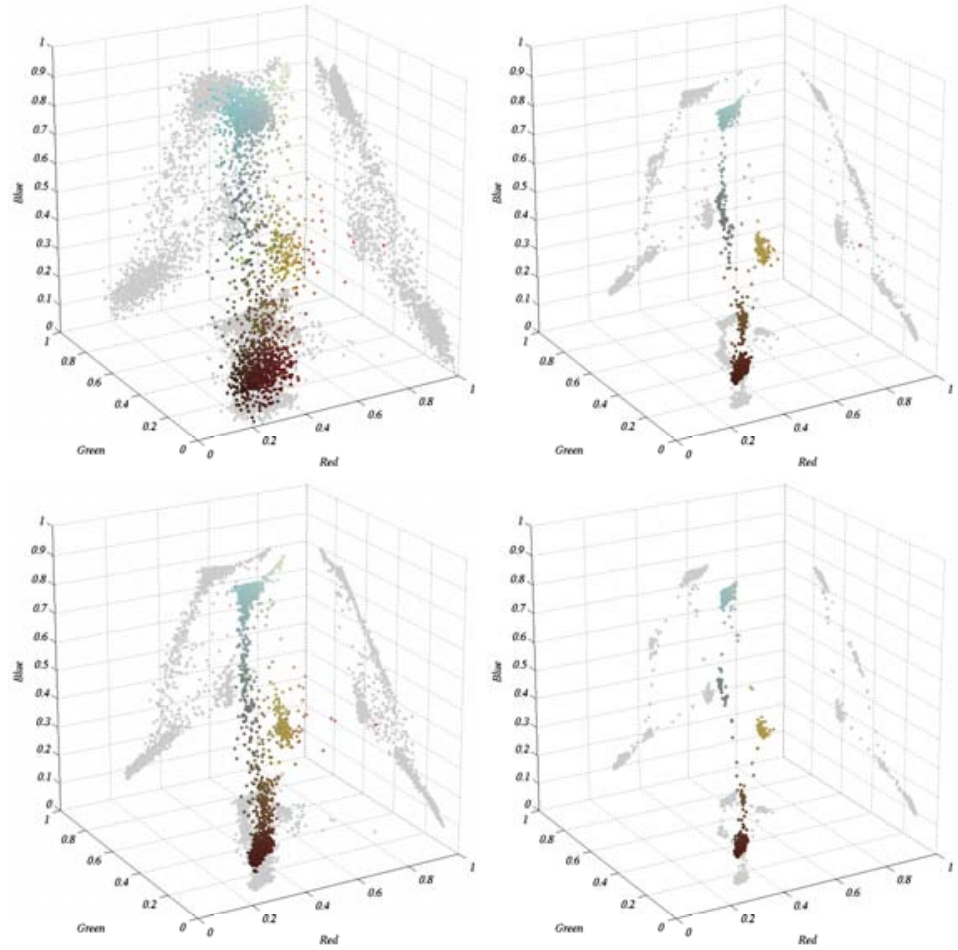

Fig. 6. Filtering unordered 3-D data. The data is the color values from Fig. 4 T-L: Noisy data. T-R: $\sigma_{v}=0.05, \sigma_{n}=0.05, \alpha=10$. B-L: $\sigma_{v}=0.05, \sigma_{n}=0.1, \alpha=0$. B-R: $\sigma_{v}=0.05$, $\sigma_{n}=0.05, \alpha=20$.

\section{Conclusion}

We have presented a novel computational framework extending the previous method proposed in [17] from scalar to vector-valued images and data. The two implementations we have presented, for images and unordered data, are examples of stochastic and deterministic variants of the framework.

While the statistical modelling used here is quite simple, it should be noted that more sophisticated Bayesian modelling could be used within the same framework, for instance to model the noise more accurately for a specific application such as X-ray imaging or Diffusion Tensor MRI (DT-MRI).

It should also be noted that the proposed method based on importance sampling could also be useful for certain cases when images are scalar-valued and the dynamic 
range is so large that it is difficult to create histograms with the precision needed. This could be the case in computed tomography (CT).

A drawback with the method is the large number of parameters and future research will have to address this issue. Nevertheless we have found our method easy to tune and use in practice. The wide range of parameters can also be regarded as a feature since it allows the filter to change characteristics, spanning for instance both low-pass and median-like filter solutions.

\section{References}

1. Andrieu, C., Freitas, N., Doucet, A., Jordan, M.I.: An Introduction to MCMC for Machine Learning. Machine Learning (2002)

2. Borik, A.C., Huang, T.S., Munson, D.C.: A generalization of median filtering using combination of order statistics. IEEE Proceedings 71(31), 1342-1350 (1983)

3. Catte, F., Lions, P.L., Morel, J.M.: Image selective smoothing and edge detection by nonlinear diffusion. SIAM Journal on Numerical Analysis I(29), 182-193 (1992)

4. Comaniciu, D., Meer, P.: Mean shift: A robust approach toward feature space analysis. IEEE Trans. Pattern Anal. Mach. Intell. 24(5), 603-619 (2002)

5. Freeman, W.T., Adelson, E.H.: The design and use of steerable filters. IEEE Transactions on Pattern Analysis and Machine Intelligence 13(9), 891-906 (1991)

6. Godtliebsen, F., Spjøtvoll, E., Marron, J.S.: A nonlinear Gaussian filter applied to images with discontinuities. Nonparametric Statistics 8, 21-43 (1997)

7. Iba, Y.: Population Monte Carlo algorithms. Transactions of the Japanese Society for Artificial Intelligence 16(2), 279-286 (2001)

8. Isard, M., Blake, A.: Condensation - conditional density propagation for visual tracking. International Journal of Computer Vision 29(1), 5-28 (1998)

9. Knutsson, H., Wilson, R., Granlund, G.H.: Anisotropic non-stationary image estimation and its applications - Part I: Restoration of noisy images. IEEE Transactions on Communications 31(3), 388-397 (1983)

10. Lee, J.S.: Digital image smoothing and the sigma filter. Computer Vision, Graphics and Image Processing 24, 255-269 (1983)

11. Liu, J.S., Chen, R., Logvienko, T.: A Theoretical Framework for Sequential Importance Sampling and Resampling. In: Doucet, A., de Freitas, N., Gordon, N. (eds.) Sequential Monte Carlo Methods in Practice, Springer, Heidelberg (2001)

12. Mrázek, P., Weickert, J., Bruhn, A.: On robust estimation and smoothing with spatial and tonal kernels. In: Klette, R., Kozera, R., Noakes, L., Weickert, J. (eds.) Geometric properties for incomplete data, pp. 335-352. Springer, Heidelberg (2006)

13. Perona, P., Malik, J.: Scale space and edge diffusion using anisotropic diffusion. IEEE Transactions on Pattern Analysis and Machine Intelligence 12(7), 629-639 (1990)

14. Rudin, L.I., Osher, S., Fatemi, E.: Nonlinear total variation based noise removal algorithms. Physica D. 60, 259-268 (1992)

15. Smith, S., Brady, J.: SUSAN - a new approach to low level image processing. International Journal of Computer Vision 23(1), 45-78 (1997)

16. Tomasi, C., Manduchi, R.: Bilateral filtering for gray and color images. In: IEEE International Conference on Computer Vision 98, pp. 839-846, Bombay, India, January 1998. IEEE

17. Wrangsjö, A., Borga, M., Knutsson. H.: A bayesian approach to image restoration. In: IEEE International Symposium on Biomedical Imaging (ISBI'04), Arlington, USA (2004) 